\title{
Quadrotor UAV indoor localization using embedded stereo camera
}

\begin{abstract}
Localization of Small-Size Unmanned Air Vehicles (UAVs) such as the Quadrotors in Global Positioning System (GPS)-denied environment such as indoors has been done using various techniques. Most of the experiment indoors that requires localization of UAVs, used cameras or ultrasonic sensors installed indoor or applied indoor environment modification such as patching (Infra Red) IR and visual markers. While these systems have high accuracy for the UAV localization, they are expensive and have less practicality in real situations. In this paper a system consisting of a stereo camera embedded on a quadrotor UAV (QUAV) for indoor localization was proposed. The optical flow data from the stereo camera then are fused with attitude and acceleration data from our sensors to get better estimation of the quadrotor location. The quadrotor altitude is estimated using Scale Invariant Feature Transform (SIFT) Feature Stereo Matching in addition to the one computed using optical flow. To avoid latency due to computational time, image processing and the quadrotor control are processed threads and core allocation. The performance of our QUAV altitude estimation is better compared to single-camera embedded QUAVs due to the stereo camera triangulation, where it leads to better estimation of the $\mathrm{x}-\mathrm{y}$ position using optical flow when fused together.
\end{abstract}

Keyword: GPS-denied environment; Odometry; Optical flow; Quadrotors; SIFT GPU; Stereo camera 